\title{
Effect of Workplace Bullying on Nurses' Job Performance
}

\author{
${ }^{1}$ Aya El Sayed Mahmoud, ${ }^{2}$ Karema Ahmed Elsaid, ${ }^{3}$ Fawzia farouk Kamel
}

(1) Bachelor of Nursing, (2) Assist. Prof of Nursing Administration, Faculty of Nursing, Tanta

University, and (3) Assist. Prof of Nursing Administration, Faculty of Nursing -Benha University,

\begin{abstract}
:
Background: Workplace bullying is a social and organizational problem within the health care system; it has several adverse effects and closely associated with nurses' job performance. Aim :The study aimed to assess the effect of workplace bullying on nurses' job performance. Research design: Descriptive correlational design was utilized to meet the aim of this study. Setting: This study was conducted in Intensive Care Unit at Benha University Hospital. Sample: Consisted of all staff nurses (65). Tools: Data was collected by using two tools, workplace bullying questionnaire and nurses' job performance observation checklist. Results: The result showed that nearly three quarters $(73.8 \%)$ of staff nurses had moderate level of bullying and more than three quarters $(81.5 \%)$ of the staff nurses had satisfactory level of job performance Conclusion: There was no statistically significant correlation between nurses' workplace bullying and their performance. Recommendation: Nurses at all levels, including administrators and staff nurses, need to be educated to report bullying and nursing administrators need to develop policies to prevent bullying and anti-bullying reporting tools and mechanisms that allow nurses who exposed to workplace bullying to report incidents of bullying.
\end{abstract}

Key words: Bullying- Nurses' Job Performance- Workplace.

\section{Introduction:}

Workplace bullying within the healthcare industry is an ever-growing problem and leaves no new graduate or experienced nurse immune to its after-effects. The health care organizations must be aware of those organizational risk factors that might have negative association with nurses' health and performance particularly bullying at workplace (Moorhead et al., 2018).

It is an imbalance of power with repeated physical or psychological aggression to a nurse or group of nurses that do not wish to be harassed. Bullying is an intentional and repetitive assault of another nurse, or group of nurses who is unable to defend themselves (Namie\&Namie, 2014; Zych et al., 2015).
There are various factors may contribute to the escalation of the bullying process like hostile work environment, formal or informal power differences between parties, societal elements like culture and traditions and lack of organizational response. Organizational wellbeing and efficient organizational response contribute to the de-escalation of the bullying process (Olsenet al., 2017).

Workplace bullying that encountered in the workplace is negatively affects nurses' professional and social lives, resignation, dismissal, loss of income and their health. Different physical, mental, and psychosomatic symptoms can be observed among nurses who have been exposed to workplace bullying. These symptoms include the following; physical 


\section{Effect of Workplace Bullying on Nurses' Job Performance}

disorders as gastrointestinal disorders, excessive weight gain or weight loss, mental disorders as depression, anxiety, loss of motivation and hypomanic. In addition to behavioral disorders as irritability, loss of concentration, exaggerated sensibility to external stimuli, lack of emotion, and suicide (Duru et al., 2018)

Workplace bullying is a serious issue in health care organizations. Bullying, as a severe interpersonal conflict, causes the devastation of both the nurses and the whole organization. Workplace bullying affects negatively on nurses' productivity, decreased job satisfaction, job security and job performance (Park \& Ono, 2016; Terzioglu et al., 2016).

Job performance is imperative in any hospital because it is one of the key indicators of productivity and profitability. Generally, job performance is prioritized by organizations to achieve organization goals. It refers to behaviors enacted by a nurse that are designed to meet hospital objectives. Job performance is all the behaviors nurses engage in while at work and how to perform at their work. Aspects such as job-specific task proficiency, behavior related to core tasks of the job, the level of commitment to core tasks, and general work behavior are becoming important factors related to job performance (Hee\&Kamaludin, 2016).

Nurses play a vital role in the global provision of health care. The performance of health-care workers, including professional nurses, is closely linked to the productivity and quality of the care provided by health care organizations. Nursing performance is an important measure of work productivity and patient safety. At Intensive Care Unit, nurses are responsible for assessing and monitoring patients' changing conditions, coordinating their care, administering medications precisely, and communicating with the patients and their families. So, health care organizations should develop strategies that can promote their nurses' health and performance (Awases et al, 2013).

\section{Significance of the study:}

Nurses perform better when a harmonious working environment exists within and across groups of health professionals; health care environments must be safe, healing and humane, respectful of the rights, responsibilities, needs and contributions of patients, their families, nurses and all health professionals.

Workplace is a place where nurses work, workplace bullying has been identified as a common challenge affecting nursing professional performance and a common problem among nurses in many hospitals especially in Intensive Care Unit due to dealing with life threating patient. So, it may affects negatively on the quality of patient care, the effectiveness of the healthcare organization as well as the nursing profession. So this study aimed to assess effect of workplace bullying on nurses' job Performance.

\section{Aim of study}

This study aimed to assess the effect of workplace bullying on nurses' job performance.

\section{Research questions:}

1. What is the workplace bullying experienced by nurses?

2. What is nurses' reaction about reporting workplace bullying?

3. What is the level of nurses' job performance?

4. Is their relationship between nurses' workplace bullying and their job performance? 


\section{Effect of Workplace Bullying on Nurses' Job Performance}

\section{Subject and Method}

\section{Research design:}

A descriptive correlational design was used to achieve the aim of the present study.

\section{Setting:}

The study was conducted in Intensive Care Unit at Benha University Hospital that located in the second floor. Consists of three isolation room each room contains two beds and four partitions each partition contains four beds.

\section{Sample:}

Study sample consisted of all (65) staff nurses who were working in predetermined setting during the time of data collection.

\section{Tools of Data collection:}

Two tools were used for data collection namely; workplace bullying questionnaire and nurses' job performance observation checklist.

\section{Tool (I): Workplace Bullying questionnaire} It included two parts:

Part I: Personal characteristics of staff nurses as: Age, gender, marital status, years of experience, and educational qualification.

\section{Part II: Workplace bullying questionnaire} (WBI) it was developed by the investigator based on Fapohunda, (2013), El-Sayed, (2015) and Lee et al., (2016) it was used to asses workplace bulling among staff nurses. It contained 96 items divided into five parts; 1Delineation of bully (1 item) that describes the bullying person, 2- Bullying acts included 35 items divided into five domains; professional threat (9 items), personal threat (11 items),work isolation (4 items), work overload (3 items), work instability (8 items). 3- Organizational system toward workplace bullying (29 items).

4- Bullying report (4 items). 5- Consequences of bullying (27 items) divided into two domains, health problem of bullying (11 items), and responses from bullying and job consequences (16 items).

\section{Scoring system}

Staff nurses' responses for delineation of bully items was either (1) for "yes" or (0) for "no". Bullying acts was measured on a three point Likert Scale ranging from (1) for never to (3) for always. Total scores were categorized into three levels as the following: Not Bullied: $<30 \%$ of total score $=<31$ point Moderate: $30-60 \%$ of total score $=31-63$ points -Severe: $>60 \%$ of total score $=>63$ points. Organizational system toward workplace bullying was measured on a five point Likert Scale ranging from (1) strongly disagree to (5) strongly agree. Responses to report and consequences of bullying items were either (1) for "yes" or (0) for "no".

Tool II: Nurses' job performance Observation Checklist It was adapted from (Youssif et al., 2017) and modified by the investigator. It aimed to assess staff nurses' job performance. It includes 54 items divided into 9 dimensions as follows: 1- Work habits (7items). 2- Staff relations and communication (8 items). 3- Communication with patients (7 items). 4Nursing care plan activities (7 items). 5Material planning and coordination (2 items). 6Safety measures and patient safety (7 items).7Innovation (2 items). 8- Documentation (9 items). 9- Keeping up-to-date technically (5 items).

\section{Scoring system:}

Staff nurses' job performance was measured on a three point Likert Scale, ranging from (0) for not applicable to (2) for done. Total scores 


\section{Aya El Sayed Mahmoud, Karema Ahmed Elsaid, Fawzia farouk Kamel}

were categorized into three levels as the following:

- Unsatisfactory: $<60 \%$ of total score $=<65$ points

- Moderate : 60 - $<75 \%$ of total score $=65-80$ points

- Satisfactory: $\geq 75 \%$ of total score $\geq 81$ points

\section{Pilot study:}

Pilot study was conducted in June 2018 on $10 \%$ of the staff nurses $(n=7)$ from total study sample, after the development of the tools and before starting data collection to determine the applicability and clarity of the tools. It helps to identify potential obstacles and problems that may encountered during period of data collection. It also, served to estimate time needed to fill the questionnaire. Nurses included in pilot study were excluded from the total study sample. Data obtained from pilot study were analyzed.

\section{Tools validity:}

Face and content of study tools were validated by jury group consisted of five experts from Nursing Administration and Psychiatric Nursing Departments from Faculties of Nursing (two Assistant Professors of Nursing Administration from Tanta University, two Assistant Professors of Nursing Administration and one Assistant Professors of Psychiatric Nursing from Menofia University). Minor modifications were done based on jury opinions.

\section{Reliability of tools}

It was measured using Cronbach's Alpha. Workplace bullying act was 0.89 . Organizational system toward workplace bullying was 0.87 . Consequences of bullying were 0.85 . Nurses' job Performance was 0.92 .

\section{Ethical Considerations}

Before conducting the study, explanation of the nature and aim of the study have been explained to staff nurses in included in the study. All subjects were informed that participation in the study is voluntary and oral consent was obtained from each participant in the study. Confidentiality of the collected data maintained. Subjects were informed that the content of the tools will be used for the research purpose only. Participants' right to withdraw from the study at any time was ascertained

\section{Preparatory phase:}

This phase started from March to May 2018. It included the following:

Reviewing the national and international related literature using journals, periodicals, textbooks, internet and theoretical knowledge of various aspects concerning the topic of the study for modifying the tools and translating the tool into Arabic language and back translation to check its accuracy.

\section{Field Work:}

The actual field work started from the beginning of July 2018 to the end of September 2018 for collecting data. The Workplace Bullying questionnaire distributed to nurses individually in their work settings, and the time needed to complete the sheet ranged from 25-35 minutes. As regards to the nurses' job performance observation checklist, the investigator observed each staff nurse during her/his work three times at different days during morning and afternoon shifts. Each nurse took from 90 to 120 minutes in each observation. The daily observed nurses ranged from 5 - 6 nurses and between the observation and the other is a month. 


\section{Effect of Workplace Bullying on Nurses' Job Performance}

\section{Results}

Table (1): shows that majority $(86.2 \%)$ of staff nurses had age from 20 to 30 years old with Mean \pm SD $(26.29 \pm 4.18)$. The majority $(90.8 \%$ and $87.7 \%$ ) of staff nurses were female and married respectively. Regarding to educational qualification, nearly half $(49.2 \%)$ of staff nurses had Technical Nursing Institute. Regarding to experience years, more than half $(53.8 \%)$ of staff nurses had less than 5 years of experience with Mean \pm SD $(4.38 \pm 2.29)$.

Figure (1) shows that more than one third $(35.3 \%)$ of staff nurses reported that bullying person was related to patients' relatives.

Table (2) shows that nearly two- thirds (64.6\%) of staff nurses reported that the bully never attempts to humiliate them in front of colleagues. More than two third $(66.2 \%$ and $67.7 \%$ ) of them reported that the bully sometimes continues to criticize and monitor their work persistently and the bully questioned their work excessively, respectively. In addition to, more than half $(55.4 \%$ and $53.8 \%)$ of them reported that they always feel confident in themselves at work and still enjoy on work, respectively.

Table (3) reveals that more than two third $(69.2 \%$ and $69.2 \%)$ of staff nurses reported that the bully never undermine their personal safety and bully makes inappropriate jokes about them. More than two-thirds $(64.6 \%)$ of them reported that they sometimes find it hard to concentrate. In addition to, more than one third $(36.9 \%)$ of them always try to avoid working with the bully.

Table (4) illustrates that more than two thirds $(66.2 \%)$ of staff nurses reported that the bully never refuses the applications of their training.
More than two fifth $(43.0 \%)$ of them reported that the bully sometimes ignores their opinions. In addition to, more than sixty (16.9\%) of them reported that the bully always withholds necessary information from them.

Table (5) shows that more than half $(58.5 \%)$ of staff nurses reported that the bully never costs them with work that don't suit them. More than half $(56.9 \%)$ of them reported that the bully sometimes sets impossible deadlines to produce work. In addition, more than fifth $(21.5 \%)$ of them reported that the bully always uses undue pressure to produce work.

Table (6) reveals that more than half $(52.3 \%)$ of staff nurses reported that the bully never removes the areas of responsibility without consultation. More than half $(58.5 \%)$ of them reported that the bully sometimes attempts to demoralize them. In addition, more than quarter $(27.7 \%)$ of them reported that always hate their career and hope the day of work to be end.

Figure (2) shows that nearly three quarters $(73.8 \%)$ of staff nurses had moderate level of bullying

Figure (3) shows that more than half $(53.8 \%)$ of staff nurses reported the bullying they faced.

Table (11) reveals that work habits of the staff nurses in the first observation majority $(90.8 \%)$ of them adheres to work schedules, in the second and third observation the majority $(95.4 \%$ and $90.8 \%)$ of them follows the applicable laws, rules, policies, and directives. While, the highest percent (53.8\% and 36.9\%) of them not completes assignments on time and not available during crunch times to work extra hours in first, second and third observation respectively.

Table (12)reveals that in the staff relations and communication of the staff nurses, the highest percent $(67.7 \%$ and $80.0 \%)$ of staff nurses collaborates with their colleges to achieve the 


\section{Aya El Sayed Mahmoud, Karema Ahmed Elsaid, Fawzia farouk Kamel}

unit objectives in the first, second and third observation, respectively. While, the highest percent $(40.0 \%, 52.3 \%$ and $40.0 \%)$ of them not sharing voice in matters of work unit in first, second and third observation, respectively.

Table (13) reveals that the staff nurses communication with patient in the first observation majority $(93.8 \%)$ of them keep confidential information of patients and highest percent $(90.8 \%$ and $95.4 \%)$ of staff nurses calm and gentle during patient care activities in second and third observation, respectively. While, the highest percent $(86.2 \%, 80.0 \%$ and $75.4 \%$ ) of staff nurses not explain the plan of care to patients in first, second and third observation, respectively.

Table (14) reveals that nursing care plan and material planning of the staff nurses, the heights percent $(100.0 \%$ and $95.4 \%)$ of staff nurses collect subjective and objective data about the patient, correct define nursing diagnosis based on assessment data and prescribe suitable intervention clearly in the first, second and third observation, respectively. While, the highest percent (15.4\% and 20.0\%) not assesse needs for equipment, materials, and processing in first and second observation, respectively and nearly one fifth $(18.5 \%)$ of them not evaluate the patient's response to intervention in third observation.

Table (15) reveals that regarding safety measures and patient safety the highest percent $(93.8 \%, 96.9 \%$ and $98.5 \%)$ of staff nurses organized work site in such a way that it is neat and clear of potential hazards in first, second and third observation, respectively. While, the highest percent $(70.8 \%, 64.6 \%$ and $60.0 \%$ ) of staff nurses not washed hands before and after any procedure in first, second and third observation, respectively.

Table (16) reveals that all (100.0\%) of staff nurses written in ink during first, second and third observation respectively. While, the highest percent $(43.1 \%, 58.5 \%$ and $53.8 \%)$ of staff nurses not Documented everything on time during first, second and third observation, respectively

Table (17) reveals that regarding keeping up-todate technically the highest percent $(46.2 \%$ and $52.3 \%$ ) of staff nurses skillful in procedures specific to the work area in the first and third observation, respectively and nearly half $(47.7 \%)$ of them served as a "resource person" on whom others rely for technical advice and knowledgeable about common diseases in work area in the second observation. While, the highest percent $(90.8 \%)$ of staff nurses not exhibited creativity in new or improved methods in the first, second and third observation, respectively

Figure (4) shows that more than three quarters $(81.5 \%)$ of the staff nurses had satisfactory level of job performance.

Figure (5) shows that there was no statistical significant correlation between nurses' overall bullying and their overall performance. 
Table (1): Frequency distribution of staff nurses according to personal characteristics $(n=65)$

\begin{tabular}{|c|c|c|}
\hline Personal characteristics & No & $\%$ \\
\hline \multicolumn{3}{|l|}{ Age (years) } \\
\hline $20-30$ & 56 & 86.2 \\
\hline $31-40$ & 6 & 9.2 \\
\hline$>40$ & 3 & 4.6 \\
\hline Mean \pm SD & \multicolumn{2}{|c|}{$26.29 \pm 4.18$} \\
\hline \multicolumn{3}{|l|}{ Gender } \\
\hline Male & 6 & 9.2 \\
\hline Female & 59 & 90.8 \\
\hline \multicolumn{3}{|l|}{ Marital status } \\
\hline Single & 8 & 12.3 \\
\hline Married & 57 & 87.7 \\
\hline \multicolumn{3}{|l|}{ Educational qualification } \\
\hline Diploma in Nursing & 8 & 12.3 \\
\hline Technical Nursing Institute & 32 & 49.2 \\
\hline Bachelor in Nursing & 25 & 38.5 \\
\hline \multicolumn{3}{|l|}{ Experience years } \\
\hline$<5$ & 35 & 53.8 \\
\hline $5-10$ & 30 & 46.2 \\
\hline Mean \pm SD & \multicolumn{2}{|c|}{$4.38 \pm 2.29$} \\
\hline
\end{tabular}

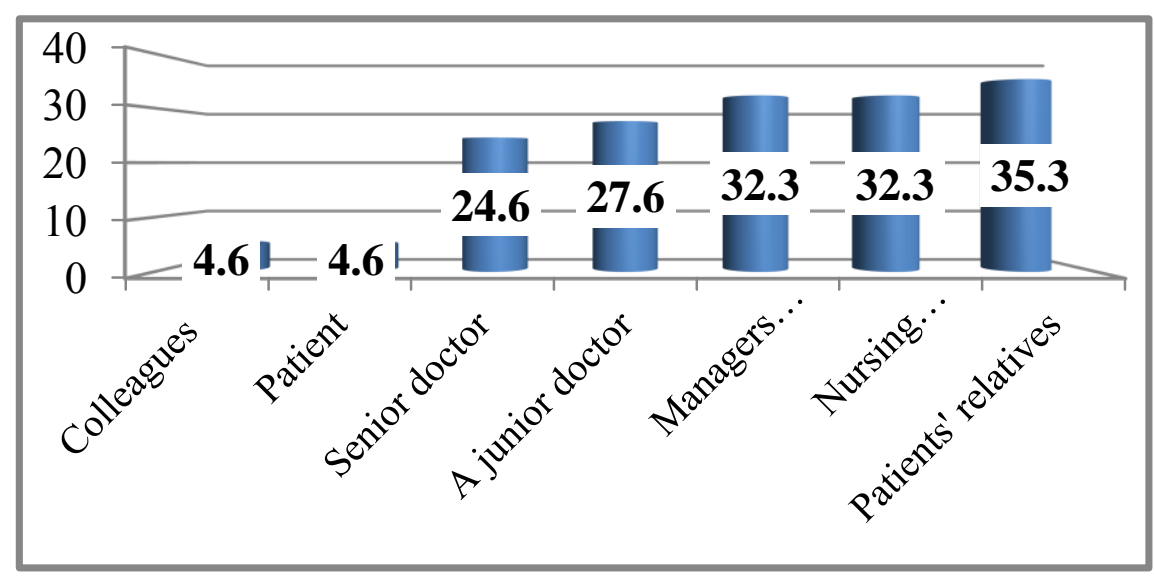

Figure (1): Frequency distribution of bullying person as reported by staff nurse 
Aya El Sayed Mahmoud, Karema Ahmed Elsaid, Fawzia farouk Kamel

Table (2): Frequency distribution of staff nurses opinion to professional threat as domain of bullying acts $(\mathbf{n}=65)$

\begin{tabular}{|l|l|l|l|l|l|l||}
\hline \multirow{2}{*}{ Professional threat } & \multicolumn{5}{|c|}{ Staff nurses' response } \\
\cline { 2 - 8 } & \multicolumn{2}{|l|}{ Always } & \multicolumn{2}{|l|}{ Sometimes } & \multicolumn{2}{|l|}{ Never } \\
\cline { 2 - 8 } & No & \% & No & \% & No & \multicolumn{1}{|c||}{} \\
\hline The bully attempts to undermine the work persistently & 9 & 13.8 & 39 & 60.0 & 17 & 26.2 \\
\hline $\begin{array}{l}\text { The bully continues to criticize and monitor the work } \\
\text { persistently }\end{array}$ & 8 & 12.3 & 43 & 66.2 & 14 & 21.5 \\
\hline The bully attempts to humiliation in front of colleagues & 0 & 0.0 & 23 & 35.4 & 42 & 64.6 \\
\hline The bully intimidation in discipline procedures & 11 & 16.9 & 28 & 43.1 & 26 & 40.0 \\
\hline The bully questioned the work excessively & 6 & 9.2 & 44 & 67.7 & 15 & 23.1 \\
\hline Going to have a good day on the way to work & 16 & 24.6 & 18 & 27.7 & 31 & 47.7 \\
\hline Trying to hide at work and be less visible & 9 & 13.8 & 24 & 36.9 & 32 & 49.3 \\
\hline Feeling confident at work & 36 & 55.4 & 23 & 35.4 & 6 & 9.2 \\
\hline Stilling enjoy on work & 35 & 53.8 & 26 & 40.0 & 4 & 6.2 \\
\hline
\end{tabular}

Table (3): Frequency distribution of staff nurses opinion to personal threat as domain of bullying acts $(\mathbf{n}=65)$

\begin{tabular}{|l|c|c|c|c|c|c||}
\hline \multirow{2}{*}{ Personal threat } & \multicolumn{4}{|c|}{ Staff nurses' response } \\
\cline { 2 - 7 } & \multicolumn{2}{|c|}{ Always } & \multicolumn{2}{c|}{ Sometimes } & \multicolumn{2}{c|}{ Never } \\
\cline { 2 - 8 } & No & \% & No & $\%$ & No & $\%$ \\
\hline The bully undermine the personal safety & 4 & 6.2 & 16 & 24.6 & 45 & 69.2 \\
\hline The bully trying to sarcasm & 4 & 6.2 & 26 & 40.0 & 35 & 53.8 \\
\hline The bully threating verbal and non-verbal & 0 & 0.0 & 26 & 40.0 & 39 & 60.0 \\
\hline The bully makes inappropriate jokes & 3 & 4.6 & 17 & 26.2 & 45 & 69.2 \\
\hline The bully trying to tease Persistently & 18 & 27.7 & 36 & 55.4 & 11 & 16.9 \\
\hline The bully blaming when problem occur from others & 13 & 20.0 & 28 & 43.1 & 24 & 36.9 \\
\hline Frightening from the bully to harm the family & 13 & 20.0 & 22 & 33.8 & 30 & 46.2 \\
\hline Feeling tired to sleep at night & 11 & 16.9 & 13 & 20.0 & 41 & 63.1 \\
\hline Find it hard to concentrate & 7 & 10.8 & 42 & 64.6 & 16 & 24.6 \\
\hline The heart beat increase when see the bully & 7 & 10.8 & 24 & 36.9 & 34 & 52.3 \\
\hline Try to avoid working with the bully & 24 & 36.9 & 16 & 24.6 & 25 & 38.5 \\
\hline
\end{tabular}


Effect of Workplace Bullying on Nurses' Job Performance

Table (4): Frequency distribution of staff nurses opinion to work isolation as domain of bullying acts $(n=65)$

\begin{tabular}{|l|c|c|c|c|c|c||}
\hline \multirow{2}{*}{ Work isolation } & \multicolumn{4}{|c|}{ Staff nurses' response } \\
\cline { 2 - 8 } & \multicolumn{2}{|c|}{ Always } & \multicolumn{2}{|c|}{ Sometimes } & \multicolumn{2}{|c|}{ Never } \\
\cline { 2 - 7 } & No & $\%$ & No & $\%$ & No & $\%$ \\
\hline The bully with holds necessary information & 11 & 16.9 & 24 & 36.9 & 30 & 46.2 \\
\hline The bully ignores the opinions & 7 & 10.8 & 28 & 43.0 & 30 & 46.2 \\
\hline $\begin{array}{l}\text { The bully denies in career development } \\
\text { opportunities }\end{array}$ & 10 & 15.4 & 19 & 29.2 & 36 & 55.4 \\
\hline The bully refuses the applications of training & 3 & 4.6 & 19 & 29.2 & 43 & 66.2 \\
\hline
\end{tabular}

Table (5): Frequency distribution of staff nurses opinion to work overload as domain of bullying acts as reported by staff nurses $(n=65)$

\begin{tabular}{|c|c|c|c|c|c|c|}
\hline \multirow{3}{*}{ Work overload } & \multicolumn{6}{|c|}{ Staff nurses' response } \\
\hline & \multicolumn{2}{|c|}{ Always } & \multicolumn{2}{|c|}{ Sometimes } & \multicolumn{2}{|c|}{ Never } \\
\hline & No & $\%$ & No & $\%$ & No & $\%$ \\
\hline The bully uses Undue pressure to produce work & 14 & 21.5 & 23 & 35.4 & 28 & 43.1 \\
\hline $\begin{array}{l}\text { The bully sets impossible deadlines to produce } \\
\text { work }\end{array}$ & 4 & 6.2 & 37 & 56.9 & 24 & 36.9 \\
\hline The bully costs with work that don't suit & 7 & 10.8 & 20 & 30.7 & 38 & 58.5 \\
\hline
\end{tabular}

Table (6): Frequency distribution of staff nurses opinion to work instability as domain of bullying acts $(\mathbf{n}=65)$

\begin{tabular}{|l|c|c|c|c|c|c||}
\hline \multirow{2}{*}{ Work instability } & \multicolumn{2}{c|}{ Always } & \multicolumn{2}{c|}{ Sometimes } & \multicolumn{2}{c|}{ Never } \\
\cline { 2 - 8 } & No & \% & No & \% & No & \% \\
\hline \hline The bully shifts goalposts without telling & 7 & 10.8 & 35 & 53.8 & 23 & 35.4 \\
\hline $\begin{array}{l}\text { The bully undervalues Constantly of the } \\
\text { efforts }\end{array}$ & 14 & 21.6 & 32 & 49.2 & 19 & 29.2 \\
\hline The bully attempts to demoralizing & 7 & 10.8 & 38 & 58.5 & 20 & 30.7 \\
\hline $\begin{array}{l}\text { The bully removes the areas of } \\
\text { responsibility without consultation }\end{array}$ & 7 & 10.8 & 24 & 36.9 & 34 & 52.3 \\
\hline The sanity begun to doubt & 7 & 10.8 & 6 & 9.2 & 52 & 80.0 \\
\hline Family and friends encourage to resign & 7 & 10.8 & 9 & 13.8 & 49 & 75.4 \\
\hline $\begin{array}{l}\text { Hate my career and hope the day of work } \\
\text { to be end }\end{array}$ & 18 & 27.7 & 23 & 35.4 & 24 & 36.9 \\
\hline $\begin{array}{l}\text { Don't try to be involved in things } \\
\text { anymore }\end{array}$ & 7 & 10.8 & 31 & 47.7 & 27 & 41.5 \\
\hline
\end{tabular}




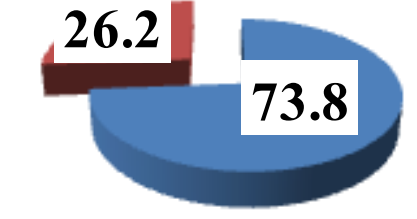

Moderate Severe

Figure (2): Overall bullying levels among the staff nurses

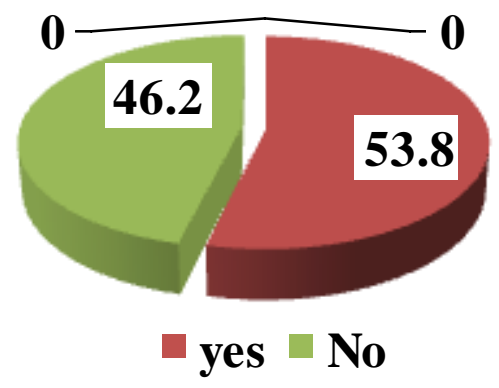

Figure (3): Reporting bullying by nurses

Table (11): Frequency distribution of staff nurses regarding work habits as domain of nurses' job performance $(n=65)$

\begin{tabular}{|c|c|c|c|c|c|c|c|c|c|c|c|c|}
\hline \multirow{3}{*}{ Work habits } & \multicolumn{4}{|c|}{ First observation } & \multicolumn{4}{|c|}{ Second observation } & \multicolumn{4}{|c|}{ Third observation } \\
\hline & \multicolumn{2}{|c|}{ Done } & \multicolumn{2}{|c|}{ Not done } & \multicolumn{2}{|c|}{ Done } & \multicolumn{2}{|c|}{ Not done } & \multicolumn{2}{|c|}{ Done } & \multicolumn{2}{|c|}{ Not done } \\
\hline & No & $\%$ & No & $\%$ & No & $\%$ & No & $\%$ & No & $\%$ & No & $\%$ \\
\hline $\begin{array}{l}\text { Follows the applicable } \\
\text { laws, rules, policies, } \\
\text { and directives. }\end{array}$ & 58 & 89.2 & 7 & 10.8 & 62 & 95.4 & 3 & 4.6 & 59 & 90.8 & 6 & 9.2 \\
\hline $\begin{array}{l}\text { Accepts instructions } \\
\text { related to her work. }\end{array}$ & 40 & 61.5 & 25 & 38.5 & 48 & 73.8 & 17 & 26.2 & 48 & 73.8 & 17 & 26.2 \\
\hline $\begin{array}{l}\text { Adheres to work } \\
\text { schedules }\end{array}$ & 59 & 90.8 & 6 & 9.2 & 56 & 86.2 & 9 & 13.8 & 56 & 86.2 & 9 & 13.8 \\
\hline $\begin{array}{l}\text { Completes } \\
\text { assignments on time }\end{array}$ & 33 & 50.8 & 32 & 49.2 & 41 & 63.1 & 24 & 36.9 & 41 & 63.1 & 24 & 36.9 \\
\hline $\begin{array}{l}\text { Responds to } \\
\text { unscheduled requests } \\
\text { on a timely basis }\end{array}$ & 31 & 47.7 & 34 & 52.3 & 42 & 64.6 & 23 & 35.4 & 42 & 64.6 & 23 & 35.4 \\
\hline $\begin{array}{l}\text { Is available during } \\
\text { crunch times to work } \\
\text { extra hours. }\end{array}$ & 30 & 46.2 & 35 & 53.8 & 41 & 63.1 & 24 & 36.9 & 41 & 63.1 & 24 & 36.9 \\
\hline $\begin{array}{l}\text { Takes responsibility } \\
\text { for his/her own work. }\end{array}$ & 40 & 61.5 & 25 & 38.5 & 45 & 69.2 & 20 & 30.8 & 45 & 69.2 & 20 & 30.8 \\
\hline
\end{tabular}


Effect of Workplace Bullying on Nurses' Job Performance

Table (12): Frequency distribution of staff nurses regarding staff relations and communication as domain of nurses' job performance $(n=65)$

\begin{tabular}{|c|c|c|c|c|c|c|c|c|c|c|c|c|}
\hline \multirow{3}{*}{$\begin{array}{l}\text { Staff relations and } \\
\text { communication }\end{array}$} & \multicolumn{4}{|c|}{ First observation } & \multicolumn{4}{|c|}{ Second observation } & \multicolumn{4}{|c|}{ Third observation } \\
\hline & \multicolumn{2}{|c|}{ Done } & \multicolumn{2}{|c|}{ Not done } & \multicolumn{2}{|c|}{ Done } & \multicolumn{2}{|c|}{ Not done } & \multicolumn{2}{|c|}{ Done } & \multicolumn{2}{|c|}{ Not done } \\
\hline & No & $\%$ & No & $\%$ & No & $\%$ & No & $\%$ & No & $\%$ & No & $\%$ \\
\hline $\begin{array}{l}\text { Communicates in a } \\
\text { good manner with all } \\
\text { staff }\end{array}$ & 37 & 56.9 & 28 & 43.1 & 45 & 69.2 & 20 & 30.8 & 48 & 73.8 & 17 & 26.2 \\
\hline $\begin{array}{l}\text { Respects all } \\
\text { members of the } \\
\text { health care team }\end{array}$ & 43 & 66.2 & 22 & 33.8 & 45 & 69.2 & 20 & 30.8 & 48 & 73.8 & 17 & 26.2 \\
\hline $\begin{array}{l}\text { Collaborates with } \\
\text { colleges to achieve } \\
\text { the unit objectives }\end{array}$ & 44 & 67.7 & 21 & 32.3 & 52 & 80.0 & 13 & 20.0 & 52 & 80.0 & 13 & 20.0 \\
\hline $\begin{array}{l}\text { Follows the ethical } \\
\text { guidelines in } \\
\text { communicating with } \\
\text { the healthcare team }\end{array}$ & 41 & 63.1 & 24 & 36.9 & 49 & 75.4 & 16 & 24.6 & 49 & 75.4 & 16 & 24.6 \\
\hline $\begin{array}{l}\text { Sharing voice in } \\
\text { matters of work unit }\end{array}$ & 31 & 47.7 & 34 & 52.3 & 39 & 60.0 & 26 & 40.0 & 42 & 64.6 & 23 & 35.4 \\
\hline $\begin{array}{ll}\text { Provide } & \text { complete } \\
\text { information } & \end{array}$ & 41 & 63.1 & 24 & 36.9 & 47 & 72.3 & 18 & 27.7 & 44 & 67.7 & 21 & 32.3 \\
\hline $\begin{array}{ll}\begin{array}{l}\text { Provide } \\
\text { information }\end{array} & \text { Reliable } \\
\end{array}$ & 41 & 63.1 & 24 & 36.9 & 47 & 72.3 & 18 & 27.7 & 44 & 67.7 & 21 & 32.3 \\
\hline $\begin{array}{ll}\text { Provide } & \text { Prompt } \\
\text { information } & \end{array}$ & 41 & 63.1 & 24 & 36.9 & 47 & 72.3 & 18 & 27.7 & 44 & 67.7 & 21 & 32.3 \\
\hline
\end{tabular}

Table (13): Frequency distribution of staff nurses regarding communication with patients as domain of nurses' job performance $(n=65)$

\begin{tabular}{|c|c|c|c|c|c|c|c|c|c|c|c|c|}
\hline \multirow{3}{*}{$\begin{array}{l}\text { Communication with } \\
\text { patients }\end{array}$} & \multicolumn{4}{|c|}{ First observation } & \multicolumn{4}{|c|}{ Second observation } & \multicolumn{4}{|c|}{ Third observation } \\
\hline & \multicolumn{2}{|c|}{ Done } & \multicolumn{2}{|c|}{ Not done } & \multicolumn{2}{|c|}{ Done } & \multicolumn{2}{|c|}{ Not done } & \multicolumn{2}{|c|}{ Done } & \multicolumn{2}{|c|}{ Not done } \\
\hline & No & $\%$ & No & $\%$ & No & $\%$ & No & $\%$ & No & $\%$ & No & $\%$ \\
\hline $\begin{array}{l}\text { Respects the dignity of } \\
\text { each patient }\end{array}$ & 54 & 83.1 & 11 & 16.9 & 52 & 80.0 & 13 & 20.0 & 58 & 89.2 & 7 & 10.8 \\
\hline $\begin{array}{l}\text { Keeps confidential } \\
\text { information of patients }\end{array}$ & 61 & 93.8 & 4 & 6.2 & 58 & 89.2 & 7 & 10.8 & 57 & 87.7 & 8 & 12.3 \\
\hline $\begin{array}{l}\text { Responds to patient's } \\
\text { needs on time. }\end{array}$ & 41 & 63.1 & 24 & 36.9 & 45 & 69.2 & 20 & 30.8 & 48 & 73.8 & 17 & 26.2 \\
\hline $\begin{array}{l}\text { Is alerts to verbal and } \\
\text { non-verbal responses }\end{array}$ & 51 & 78.5 & 14 & 21.5 & 49 & 75.4 & 16 & 24.6 & 55 & 84.6 & 10 & 15.4 \\
\hline $\begin{array}{l}\text { Is calms and gentle } \\
\text { during patient care } \\
\text { activities }\end{array}$ & 58 & 89.2 & 7 & 10.8 & 59 & 90.8 & 6 & 9.2 & 62 & 95.4 & 3 & 4.6 \\
\hline $\begin{array}{l}\text { Behaves ethically and } \\
\text { politely with patients }\end{array}$ & 51 & 78.5 & 14 & 21.5 & 55 & 84.6 & 10 & 15.4 & 55 & 84.6 & 10 & 15.4 \\
\hline $\begin{array}{l}\text { Explains the plan of } \\
\text { care to patients. }\end{array}$ & 9 & 13.8 & 56 & 86.2 & 13 & 20.0 & 52 & 80.0 & 16 & 24.6 & 49 & 75.4 \\
\hline
\end{tabular}


Aya El Sayed Mahmoud, Karema Ahmed Elsaid, Fawzia farouk Kamel

Table (14): Frequency distribution of staff nurses regarding nursing care plan and material planning domains of nurses' job performance $(n=65)$

\begin{tabular}{|c|c|c|c|c|c|c|c|c|c|c|c|c|}
\hline \multirow{3}{*}{ Nursing care plan } & \multicolumn{4}{|c|}{ First observation } & \multicolumn{4}{|c|}{ Second observation } & \multicolumn{4}{|c|}{ Third observation } \\
\hline & \multicolumn{2}{|c|}{ Done } & \multicolumn{2}{|c|}{ Not done } & \multicolumn{2}{|c|}{ Done } & \multicolumn{2}{|c|}{ Not done } & \multicolumn{2}{|c|}{ Done } & \multicolumn{2}{|c|}{ Not done } \\
\hline & No & $\%$ & No & $\%$ & No & $\%$ & No & $\%$ & No & $\%$ & No & $\%$ \\
\hline $\begin{array}{l}\text { Collects subjective } \\
\text { data about the patient }\end{array}$ & 65 & 100.0 & 0 & 0.0 & 62 & 95.4 & 3 & 4.6 & 62 & 95.4 & 3 & 4.6 \\
\hline $\begin{array}{l}\text { Collects objective } \\
\text { data about the patient }\end{array}$ & 65 & 100.0 & 0 & 0.0 & 65 & 100.0 & 0 & 0.0 & 62 & 95.4 & 3 & 4.6 \\
\hline $\begin{array}{l}\text { Corrects define nursing } \\
\text { diagnosis based on } \\
\text { assessment data }\end{array}$ & 65 & 100.0 & 0 & 0.0 & 65 & 100.0 & 0 & 0.0 & 62 & 95.4 & 3 & 4.6 \\
\hline $\begin{array}{l}\text { Sets priorities for } \\
\text { patient's problems. }\end{array}$ & 61 & 93.8 & 4 & 6.2 & 61 & 93.8 & 4 & 6.2 & 58 & 89.2 & 7 & 10.8 \\
\hline $\begin{array}{l}\text { Prescribes suitable } \\
\text { intervention clearly. }\end{array}$ & 65 & 100.0 & 0 & 0.0 & 65 & 100.0 & 0 & 0.0 & 62 & 95.4 & 3 & 4.6 \\
\hline 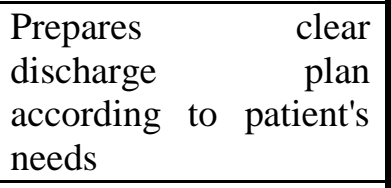 & 59 & 90.8 & 6 & 9.2 & 59 & 90.8 & 6 & 9.2 & 56 & 86.2 & 9 & 13.8 \\
\hline $\begin{array}{l}\text { Evaluates the } \\
\text { patient's response to } \\
\text { intervention }\end{array}$ & 56 & 86.2 & 9 & 13.8 & 56 & 86.2 & 9 & 13.8 & 53 & 81.5 & 12 & 18.5 \\
\hline $\begin{array}{l}\text { Material planning } \\
\text { and Coordination }\end{array}$ & & & & & & & & & & & & \\
\hline $\begin{array}{ll}\text { Assesses needs for } \\
\text { equipment, } \\
\text { materials, } \\
\text { processing }\end{array}$ & 55 & 84.6 & 10 & 15.4 & 52 & 80.0 & 13 & 20.0 & 55 & 84.6 & 10 & 15.4 \\
\hline $\begin{array}{l}\text { Utilizes available } \\
\text { support services (e.g. } \\
\text { laboratory, laundry, } \\
\text { pharmacy) } \\
\text { effectively. }\end{array}$ & 58 & 59.2 & 7 & 10.8 & 55 & 84.6 & 10 & 15.4 & 58 & 89.2 & 7 & 10.8 \\
\hline
\end{tabular}


Effect of Workplace Bullying on Nurses' Job Performance

Table (15): Frequency distribution of staff nurses regarding safety measures and patient safety as domain of nurses' job performance $(n=65)$

\begin{tabular}{|c|c|c|c|c|c|c|c|c|c|c|c|c|}
\hline \multirow{3}{*}{$\begin{array}{l}\text { Safety measures and } \\
\text { patient safety }\end{array}$} & \multicolumn{4}{|c|}{ First observation } & \multicolumn{4}{|c|}{ Second observation } & \multicolumn{4}{|c|}{ Third observation } \\
\hline & \multicolumn{2}{|c|}{ Done } & \multicolumn{2}{|c|}{ Not done } & \multicolumn{2}{|c|}{ Done } & \multicolumn{2}{|c|}{ Not done } & \multicolumn{2}{|c|}{ Done } & \multicolumn{2}{|c|}{ Not done } \\
\hline & No & $\%$ & No & $\%$ & No & $\%$ & No & $\%$ & No & $\%$ & No & $\%$ \\
\hline $\begin{array}{l}\text { Washes hands before and } \\
\text { after any procedure }\end{array}$ & 19 & 29.2 & 46 & 70.8 & 23 & 35.4 & 42 & 64.6 & 26 & 40.0 & 39 & 60.0 \\
\hline $\begin{array}{l}\text { Puts needed personal } \\
\text { protective during patient } \\
\text { care activities e.g. gloves } \\
\text { mask.....etc. }\end{array}$ & 26 & 40.0 & 39 & 60.0 & 30 & 46.2 & 35 & 53.8 & 30 & 46.2 & 35 & 53.8 \\
\hline $\begin{array}{l}\text { Applies preventive } \\
\text { measures to prevent hazards } \\
\text { according to needs }\end{array}$ & 60 & 92.3 & 5 & 7.7 & 62 & 95.4 & 3 & 4.6 & 63 & 96.9 & 2 & 3.1 \\
\hline $\begin{array}{l}\text { Follows safety measures to } \\
\text { avoid physical hazards e.g. } \\
\text { avoid bedside rails, } \\
\text { infection control guidelines. }\end{array}$ & 56 & 86.2 & 9 & 13.8 & 62 & 95.4 & 3 & 4.6 & 62 & 65.4 & 3 & 4.6 \\
\hline $\begin{array}{l}\text { Organizes work site in such } \\
\text { a way that it is neat and } \\
\text { clear of potential hazards. }\end{array}$ & 61 & 93.8 & 4 & 6.2 & 63 & 96.9 & 2 & 3.1 & 64 & 98.5 & 1 & 1.5 \\
\hline $\begin{array}{l}\text { Follows five rights of } \\
\text { medication administration }\end{array}$ & 54 & 83.1 & 11 & 16.9 & 56 & 86.2 & 9 & 13.8 & 51 & 78.5 & 14 & 21.5 \\
\hline $\begin{array}{l}\text { Seeks agreement from } \\
\text { patient before any } \\
\text { procedure }\end{array}$ & 44 & 67.7 & 21 & 32.3 & 40 & 61.5 & 25 & 38.5 & 40 & 61.5 & 25 & 38.5 \\
\hline
\end{tabular}

Table (16): Frequency distribution of staff nurses regarding documentation as domain of nurses' job performance $(n=65)$

\begin{tabular}{|c|c|c|c|c|c|c|c|c|c|c|c|c|}
\hline \multirow{3}{*}{ Documentation } & \multicolumn{4}{|c|}{ First observation } & \multicolumn{4}{|c|}{ Second observation } & \multicolumn{4}{|c|}{ Third observation } \\
\hline & \multicolumn{2}{|c|}{ Done } & \multicolumn{2}{|c|}{ Not done } & \multicolumn{2}{|c|}{ Done } & \multicolumn{2}{|c|}{ Not done } & \multicolumn{2}{|c|}{ Done } & \multicolumn{2}{|c|}{ Not done } \\
\hline & No & $\%$ & No & $\%$ & No & $\%$ & No & $\%$ & No & $\%$ & No & $\%$ \\
\hline Concise & 48 & 73.8 & 17 & 26.2 & 52 & 80.0 & 13 & 20.0 & 50 & 76.9 & 15 & 23.1 \\
\hline Organized & 60 & 92.3 & 5 & 7.7 & 62 & 95.4 & 3 & 4.6 & 63 & 96.9 & 2 & 3.1 \\
\hline Easy to read & 58 & 59.2 & 7 & 10.8 & 55 & 84.6 & 10 & 15.4 & 58 & 89.2 & 7 & 10.8 \\
\hline Written in ink & 65 & 100.0 & 0 & 0.0 & 65 & 100.0 & 0 & 0.0 & 65 & 100.0 & 0 & 0.0 \\
\hline $\begin{array}{l}\text { Documents } \\
\text { everything on time }\end{array}$ & 37 & 56.9 & 28 & 43.1 & 27 & 41.5 & 38 & 58.5 & 30 & 46.2 & 35 & 53.8 \\
\hline $\begin{array}{l}\text { Records clearly current } \\
\text { condition of patient }\end{array}$ & 49 & 75.4 & 16 & 24.6 & 52 & 80.0 & 13 & 20.0 & 52 & 80.0 & 13 & 20.0 \\
\hline $\begin{array}{l}\text { Records progress in } \\
\text { patient condition. }\end{array}$ & 48 & 73.8 & 17 & 26.2 & 53 & 81.5 & 12 & 18.5 & 53 & 81.5 & 12 & 18.5 \\
\hline $\begin{array}{l}\text { Records all } \\
\text { procedures performed }\end{array}$ & 56 & 86.2 & 9 & 13.8 & 62 & 95.4 & 3 & 4.6 & 56 & 86.2 & 9 & 13.8 \\
\hline $\begin{array}{l}\text { Provides others with } \\
\text { complete and accurate } \\
\text { written directions }\end{array}$ & 41 & 63.1 & 24 & 36.9 & 39 & 60.0 & 26 & 40.0 & 42 & 64.6 & 23 & 35.4 \\
\hline
\end{tabular}


Aya El Sayed Mahmoud, Karema Ahmed Elsaid, Fawzia farouk Kamel

Table (17): Frequency distribution of staff nurses regarding keeping up-to-date technically domain of nurses' job performance $(n=65)$

\begin{tabular}{|c|c|c|c|c|c|c|c|c|c|c|c|c|}
\hline \multirow{3}{*}{$\begin{array}{l}\text { Keeping up-to-date } \\
\text { technically }\end{array}$} & \multicolumn{4}{|c|}{ First observation } & \multicolumn{4}{|c|}{ Second observation } & \multicolumn{4}{|c|}{ Third observation } \\
\hline & \multicolumn{2}{|c|}{ Done } & \multicolumn{2}{|c|}{ Not done } & \multicolumn{2}{|c|}{ Done } & \multicolumn{2}{|c|}{ Not done } & \multicolumn{2}{|c|}{ Done } & \multicolumn{2}{|c|}{ Not done } \\
\hline & No & $\%$ & No & $\%$ & No & $\%$ & No & $\%$ & No & $\%$ & No & $\%$ \\
\hline $\begin{array}{l}\text { Serves as a "resource } \\
\text { person" on whom } \\
\text { others rely for } \\
\text { technical advice }\end{array}$ & 16 & 24.6 & 49 & 75.4 & 31 & 47.7 & 34 & 52.3 & 28 & 43.1 & 37 & 56.9 \\
\hline $\begin{array}{l}\text { Skillful in procedures } \\
\text { specific to the work } \\
\text { area }\end{array}$ & 30 & 46.2 & 35 & 53.8 & 28 & 43.1 & 37 & 56.9 & 34 & 52.3 & 31 & 47.7 \\
\hline $\begin{array}{l}\text { Knowledgeable about } \\
\text { common diseases in } \\
\text { his/her work area }\end{array}$ & 24 & 36.9 & 41 & 63.1 & 31 & 47.7 & 34 & 52.3 & 28 & 43.1 & 37 & 56.9 \\
\hline $\begin{array}{l}\text { Demonstrates effort } \\
\text { and success at self- } \\
\text { improvement. }\end{array}$ & 20 & 30.8 & 45 & 69.2 & 16 & 24.6 & 49 & 75.4 & 16 & 24.6 & 49 & 75.4 \\
\hline $\begin{array}{l}\text { Strives to achieve } \\
\text { objectives beyond } \\
\text { what is expected or } \\
\text { required }\end{array}$ & 12 & 18.5 & 53 & 81.5 & 19 & 29.2 & 46 & 70.8 & 16 & 24.6 & 49 & 75.4 \\
\hline Innovation & & & & & & & & & & & & \\
\hline Develop new solution & 9 & 13.8 & 56 & 86.2 & 9 & 13.8 & 56 & 86.2 & 12 & 18.5 & 53 & 81.5 \\
\hline $\begin{array}{l}\text { Exhibits creativity in } \\
\text { new or improved } \\
\text { methods }\end{array}$ & 6 & 9.2 & 59 & 90.8 & 6 & 9.2 & 59 & 90.8 & 6 & 9.2 & 59 & 90.8 \\
\hline
\end{tabular}

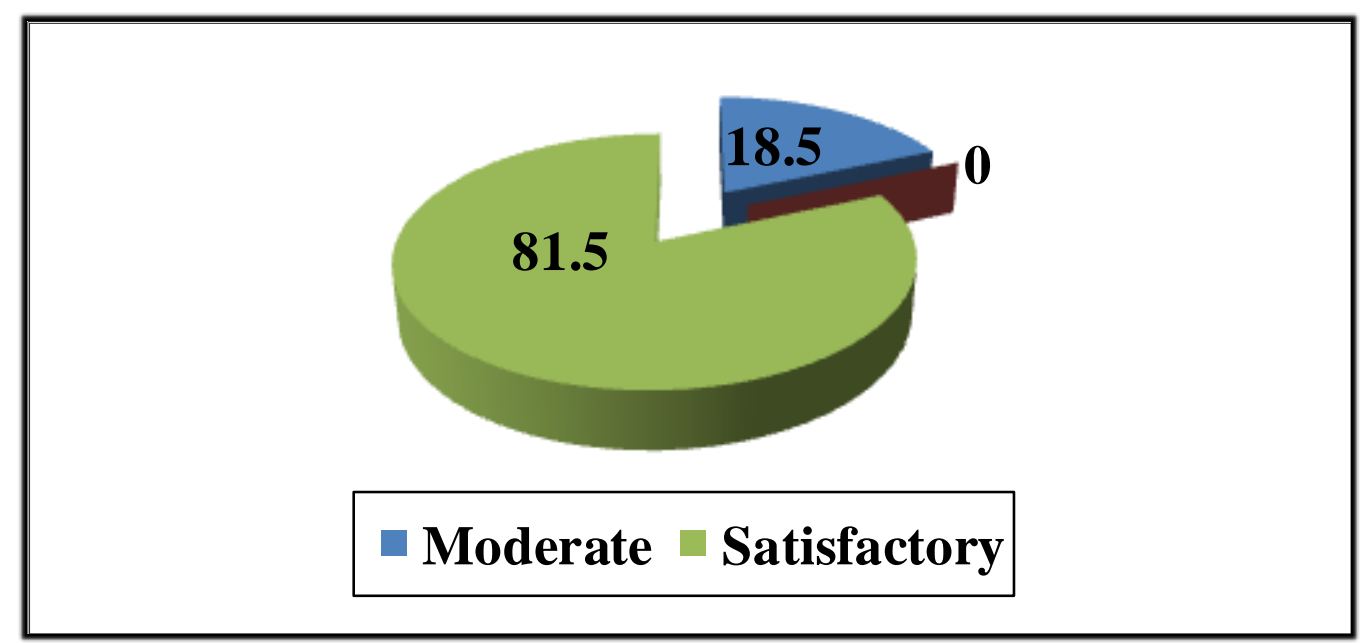

Figure (4): Frequency distribution of staff nurses according to average levels of nurses' job performance 


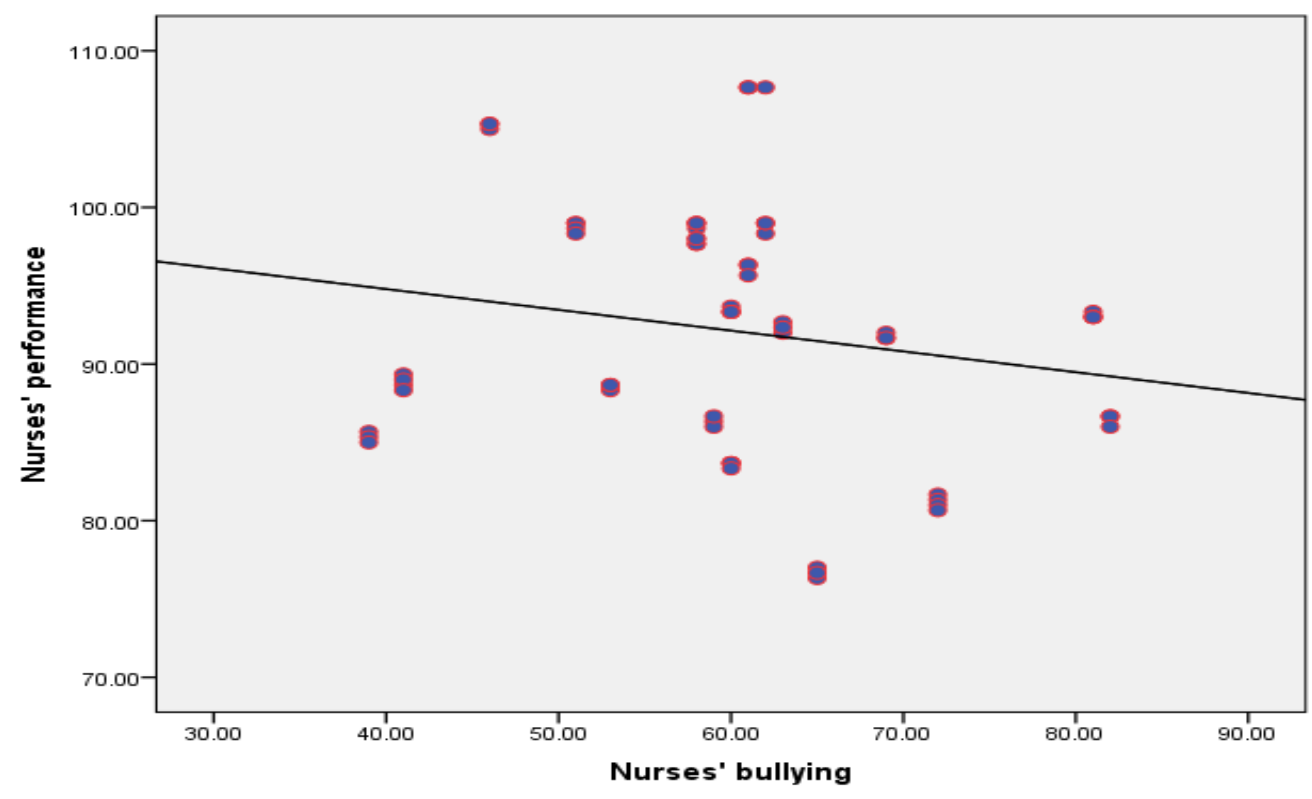

Figure (5): Correlation between nurses' overall bullying and their overall performance

\section{Discussion}

The health care organization has been identified as one of workplace bullying can occurs frequently. Bullying present in all work environments but nurses are on the frontline of the healthcare system and have the closest contact with patients and their relatives. Bullying is a serious issue affecting the nursing profession. It has serious negative consequences that may extend beyond individual nurses to an entire health care organization such as experiencing stress, frustration, physical and psychological disorders, absenteeism, poor performance and leaves a particular place of employment (Samir et al., 2012; Yamada et al., 2018).

The present study aimed to assess the effect of workplace bullying on nurses' job performance.

The result of the present study covered fifth main areas as following, firstly; nurses' bullying, secondly; nurses' job performance, thirdly; relation between bullying levels and personal characteristics of staff nurses, fourthly; relation between job performance levels and personal characteristics of staff nurses and finally; correlation between nurses' bullying and their performance.

\section{Nurses' Bullying}

Regarding bully person, the present study revealed that more than one third of staff nurses reported that bullying person was related to patients' relatives. This is due to dealing with life threatening patient conditions reflected in nervousness and tension to patients' relatives which portrayed in bullying behavior or acts.

This result in agreement with Samir et al., (2012) found that patient's relatives were the most usual source of violence against nurses, followed by members of administrative and medical teams. Also, Khoshknab et al., (2015) reported that the 


\section{Aya El Sayed Mahmoud, Karema Ahmed Elsaid, Fawzia farouk Kamel}

main source of bullying was patients' families.

On the other hand, this result contradicted with Bardakçı, (2016) reported that most of the bullying behaviors were perpetratedby head nurses followed by physicians, Berry et al., (2016) showed that the peer to-peer or mentor-to-new hire bullying occurred due to the increased workload the mentor encountered when training the apprentice nurse. Efe and Ayaz's, (2010), Cevic-Akyıl et al., (2012) and Trepanier et al., (2013) found that nurses are subjected for bully behaviors perpetrated by head nurses and physicians, Clendon and Walker, (2012) revealed that, staff nurses were exposed to bullying but the bully person was senior and older nurses. Also Abbas et al., (2010) reported that the bully person reported by nurses in their studies was mainly the patient followed by colleagues and administrative staff or supervisors committed the abuse.

In relation to professional threat as domain of bullying acts. The present study revealed that nearly two third of staff nurses reported that the bully never attempts to humiliate them in front of colleagues. More than two third of them reported that the bully sometimes continues to criticize and monitor their work persistently and the bully questioned their work excessively. In addition, more than half of them reported that always feel confident in them at work and still enjoy on work, this is due to the ability of staff nurses to deal with bullies.

This result agreed with El-Sayed, (2015) who reported that more than half of staff nurses never threatened or publicly humiliated by the bully and nearly half of them always feeling confident in themselves at work and still enjoy on work. While, this result disagreed with Nwaneri et al., (2016); Ekici and Beder, (2014) they found that more than half of staff nurses experienced bullying as being humiliated in front of others including patients.

Regarding to personal threat as domain of bullying acts the present study results revealed that more than two thirds of staff nurses reported that the bully never undermine their personal safety and bully makes inappropriate jokes about them. More than two third of them reported that they sometimes find it hard to concentrate. In addition, more than one third of them always try to avoid working with the bully. This is due to the most of the nurses felt that expression of such acts threatened their dignity so avoid working with the bully.

In the same line, El-Sayed, (2015) reported that nearly half of staff nurses regularly try and avoid working with the bullies, Gates et al., (2011) reportedthatthe nurses who had experience of bulling suffered from difficulty thinking, and decreased concentration . Also, Vessey et al., (2010) documented that physical and psychological effects of bullying causedhealth problems as inability to concentrate,. On the other hand, this result disagreed with Ekici and Beder, (2014) who found that nurses experienced bullying being wrongly blamed.

Concerning work isolation as domain of bullying acts, the present study results revealed that more than two-thirds of staff nurses reported that bully never refuses the applications of their training. More than two fifth of them reported that the bully sometimes ignores their opinions. In addition, more than sixty of them reported that the bully always withholds necessary information from them. This is due to the bully withholds 


\section{Effect of Workplace Bullying on Nurses' Job Performance}

information from nurses to make them perform poorly on their jobs.

This result is in agreement with Fapohunda, (2013) who reported that more than half of staff nurses their bullies purposely held back information from them. Also, found that nearly half of staff nurses observed that their professional opinions and views were never valued and they received hostile answers to questions.

In relation to work overload as domain of bullying acts the result of the present study revealed that more than half of staff nurses reported that the bully never costs them with work that don't suit them. The bully sometimes sets impossible deadlines to produce work. In addition, more than one fifth of them reported that the bully always uses undue pressure to produce work. This is due to the perpetrator is often senior position and the subsequent complaint may be seen as simply jealousy, resulting in repercussions and a certain amount of pressure.

The result of the present study agreed with Fapohunda, (2013) who found that more than one third of staff nurses reported that they were often assigned tasks with impossible deadlines by their bullies. Also, nearly half of them observed that their bullies use undue pressure to produce work. While, the result of the present study was inconsistent with Nwaneri et al., (2016) who reported that the bullies assigned them tasks that not suit, were clearly outside their job descriptions and unfair allocation of job and postings.

Regarding to work instability as domain of bullying acts, the result of the present study revealed that more than half of staff nurses reported that the bully never removes the areas of responsibility without consultation; the bully sometimes attempts to demoralize them. In addition, more than quarter of them reported that always hate their career and hope the day of work to be end. This is due to nurses still believe that bullying is normal behavior in workplace and many of staff members may actually be unaware that they are tolerating unacceptable behavior amongst their workplace and a misunderstanding of staff rights and responsibilities that lead to heat the career.

The result of the present study is consistent with Nwaneri et al., (2016) reported that the staff nurses hate the career and wish to leave the career to avoid the bulling, Fapohunda, (2013) who found that more than one third of the staff nurses reported that their bosses repeatedly checked their works and demoralize them even the completion of important assignments were never appreciated,.

Concerning bullying levels among the staff nurses, the present study revealed that that nearly three quarter of staff nurses had moderate level of bullying. This is due to the staff nurses communicate respectfully with each other and there is no more interaction between nurses and patient relatives. The result of the present study disagreed with Nwaneri et al., (2016) found work place bullying among nurses is reported to be high.

In relation to reporting bullying by nurses, the result of the present study revealed that more than half of the staff nurses reported the bullying. This is due to the hospital administrators constitute disciplinary committee this encourage nurses to report the incidence of workplace bullying, punish perpetrators, as well as, protect those who report the cases from further attacks of bullying through establishment of policies to regulate interpersonal relationships among nurses. 


\section{$\underline{\text { Aya El Sayed Mahmoud, Karema Ahmed Elsaid, Fawzia farouk Kamel }}$}

This result was consistent with El-Sayed, (2015) who reported that nurses make a report for bullying exposure to nursing director and head nurse. On the other hand, this result contrasted with Bardakçı, (2016) reported that the nurses reacted to bullying behaviors mostly by keeping silent and sharing the issue with friends and family, Nwaneri et al., (2016) reported that majority of the victims of workplace bullying did not report the bullying incident to a relevant authority. Also, Khoshknab et al., (2015) reported that more than half of participants did not report bullying.

Also, the result of the present study revealed that nearly one quarter of staff nurses reported that they didn't know how reporting. This result in the same line with Chesler, (2014) found that many of the nurses did not know what was done reporting, Bakker, (2012) who found that forty five of participated nurses were exposed to covert violence from management/senior staff but not report this bullying because they did not know reporting.

On the other hand, the result in contrasted with Cevic-Akyıl et al., (2012) who reported that nurses preferred to remain silent and tend to share their discontent only with friends. Also, Khoshknab et al., (2015) reported that no guidelines existed in the healthcare settings for dealing with violence, and once violent are reported, no action was taken by superiors.

\section{Nurses' job performance}

In relation to work habits of the staff nurses, the result of the present study revealed that in the first observation majority of them adheres to work schedules; in the second and third observation the majority of them follow the applicable laws, rules, policies, and directives. While, the highest percent of them did not complete assignments on time and not available during crunch times to work extra hours in first, second and third observation. From the researcher point of view, this is due to the nurse's takes responsibilities toward the work and follows the ethical consideration rules toward the working hours.

Regarding to the staff nurses relations and communication, the result of the present study revealed that the highest percent of staff nurses collaborates with their colleges to achieve the unit objectives in the first, second and third observation. While the highest percent of them did not sharing voice in matters of work unit in first, second and third observation. This is due to in intensive care unit most of patient in coma and the staff nurses cooperate with each other and more careful with dealing with life threatening patient in trying to save the patient life. This result aligned with Safarpour et al.,(2018) found that highest level of job performance was related to communication and interpersonal relations.

While, this result disagreed with Nwaneri et al., (2016) revealed that the more nurses are exposed to workplace bullying affect on the social relationships and communication among them, Ibrahim et al., (2016) found the nurses had unacceptable level of respect, communication, teamwork and collaboration with their each other, Ekici\&Beder, (2014)) who found that the negative effects of bullying that nurses experienced were a lack of communication, reduced collaboration with co-workers and managers. Also, Gates et al., (2011) who reported that nurses who had bullying experience had difficulty communicating with patients or other health care providers.

Concerning communication with patients, the present study revealed that the 


\section{Effect of Workplace Bullying on Nurses' Job Performance}

majority of staff nurses in the first observation keep confidential information of patients and highest percent of staff nurses calm and gentle during patient care activities in second and third observation. While, the highest percent of staff nurses did not explain the plan of care to patients in first, second and third observation. This is due to nurses respect the ethics of nursing profession, patient right and autonomy of the patient.

This result agreed with, Sharma and Dhar, (2016) found that nursing staff perform their duties quite well considering their ethical and moral duty during patient care activities to give the best services to patients and nurses tend to become emotionally attached to patients as well as to the organization.

In relation to nursing care plan and material planning domains of nurses' job performance. This result revealed that the highest percent of staff nurses collect subjective and objective data about the patient, correct define nursing diagnosis based on assessment data and prescribe suitable intervention clearly in the first, second and third observation. While, the highest percent not assess needs for equipment, materials, and processing in first and second observation and nearly one fifth of them not evaluate the patient's response to intervention in third observation. This is due to the nurses have the skill to collect patient data and nursing diagnosis.

This result supported by Shrestha, (2016) who reported that the nurses collect information about nursing diagnosis, planning, implementation and evaluation of the plan done to patient care in each shift. On the other hand, this result contrasted with Ibrahim et al., (2016) reported that nearly three-quarters of staff nurses had unacceptable level of nursing assessment, formulating nursing diagnoses, formulating and initiating a plan to provide appropriate care. Also, Elshahat, (2014) who stated that the nurses evaluate the patient's response to intervention.

Regarding to safety measures and patient safety the result revealed that the highest percent of staff nurses organized work site in such a way that it is neat and clear of potential hazards in first, second and third observation. While, the highest percent of staff nurses did not washed hands before and after any procedure in first, second and third observation. This is due to the majority of intensive care unit patient unconscious and more prone to several risks as patient falls and bed sores. This result disagreed with Ibrahim et al., (2016) who reported that the majority of staff nurses had unacceptable level of maintaining the patient safety as providing appropriate interventions for patients at risk of falls.

Concerning documentation as domain of nurses' job performance. This study revealed that, all of staff nurses written in ink during first, second and third observation. While the highest percent of staff nurses not documented everything on time during first, second and third observation. This is due to written in ink is the most important principles of manual documentation, for legal purposes and this allows nursing staff to acknowledge the care provided, share it to ensure continuity and reassess its effects and patient needs as required and protect the nurse legally, to act as a communication tool, to promote quality nursing care. This result disagreed with Hojat and Taheri, (2014) who found that the inappropriate performance of the caring staff in data record and document. 


\section{Aya El Sayed Mahmoud, Karema Ahmed Elsaid, Fawzia farouk Kamel}

In relation to keeping up-to-date technically, this study found that the highest percent of staff nurses skillful in procedures specific to the work area in the first and third observation and nearly half of them served as a resource person on whom others rely for technical advice and knowledgeable about common diseases in work area in the second observation. While the highest percent of staff nurses not exhibited creativity in new or improved methods in the first, second and third observation. This due to nurses work under system routines, so they do the same tasks every day.

This result disagreed with Ibrahim et al., (2016) who reported that no one of the nurses had acceptable scores pertaining to acquire and maintain current knowledge in nursing practice, maintain self-learning, and develop abilities that would ensure that they are knowledgeable on recent developments in the field.

Regarding to, the level of nurses' job performance, the result of the present study revealed that, more than three-quarters of the staff nurses had satisfactory level of job performance. This is due to the staff nurses had enough skill, suitable resources and equipment for complete the task. This result agreed with Safarpour et al., (2018) and Sumathi et al., (2013) reported that nurses had high levels of job performance.

While, this result disagreed with Ibrahim et al., (2016) indicated that the performance scores among staff nurses were relatively low, and all nurses' performance was incompetent and needs improvement, Elkholy (2015) who reported the performance of staff nurses was not good, Hojat and Taheri, (2014) found that the quality of nurses' performance was assessed as moderate level, Al-Makhaita et al., (2014) they found nurses working in intensive care unit rated lower performance than others. Also, Al-Homayan et al., (2013) who concluded that the level of staff nurses was moderate.

Correlation between staff nurses' bullying and their job performance

The result of the present study revealed that, there was no statistically significant correlation between nurses' bullying and their performance. This is due to the nurses had ability to deal with bullying, policies existed in the healthcare settings for dealing with bullying, and once bullying were reported, action was taken by superiors, nurses are able to function at their full capacity when bullying continue in the work environment, Also, good communication among nurses about patient care, positively affected the nurses' ability to deliver highquality patient care. Also, teamwork and nursing morale are not affected when the hostile behaviors occur among nursing peers.

This result disagreed with Ikyanyon\&Ucho, (2013); Mete and Sökmen, (2016); Khalique et al, (2018) found a negative correlation between workplace bullying and job performance. Fapohunda, (2013) found more than half of the staff nurses felt their work productivities and performances were negatively affected by bullying act. Also, Broeder and Donze (2010) who found that the workplace bullying impairs the nurses' professional performance.

\section{Conclusion:}

There was no statistically significant correlation between nurses' workplace bullying and their performance. 


\section{Effect of Workplace Bullying on Nurses' Job Performance}

\section{Recommendations}

In the light of the findings obtained from the present study, the following recommendations are suggested:

\section{Nursing level:}

1. Nurses at all levels, including administrators and staff nurses need to be informed them to report bullying.

2. Conducting training program for nurses about bullying and different strategies to deal with it, including verbal, nonverbal and writing down techniques.

3. Nurses need to pay attention for patients' complains through investigating patients, visitors' opinions and suggestions to avoid the bullying reaction of the patient and their relatives in the case of ignorance.

\section{Organizational level:}

4. Nursing administrators developing policies to prevent bullying and antibullying reporting tools and mechanisms that allow nurses who exposed to workplace bullying to report incidents of bullying.

5. Provide support, psychological counseling and comprehensive care for nurses' victim of bullying and inform nurses about their rights to create a positive atmosphere for nurses.

\section{Further researches are suggested}

6. Conducted study to identify the effective strategies to eliminate bullying behaviors.

7. Assess the impact of workplace bullying on the organizational outcomes.

\section{References}

Abbas, M., Fiala, L., Abdel Rahman, A. and Fahim, E. (2010). Epidemiology of workplace violence against nursing staff in Ismailia Governorate, Egypt. J Egypt Public Health Assoc., 85(1-2), 29-43. PMID: 21073846. Available at: http://www. ncbi.nlm.nih.gov/pubmed/21073846.
Al-Homayan, A. M., Shamsudin, F. M., Subramaniam, C., \& Islam, R. (2013). Impacts Of Job Demands On Nurses' performance Working In Public Hospitals. American Journal of Applied Sciences, 10(9), 1050.

Al-Makhaita, H., Sabra, A. \& Hafez, A. (2014). Job performance among nurses working in two different health care levels, Eastern Saudi Arabia: a comparative study. International Journal of Medical Science and Public Health, 3(7), 832-838.

Awases, M., Bezuidenhout, M., \&Roos, J. (2013).Factors affecting the performance of professional nurses in Namibia.curationis, 36(1), 1-8.

Bakker, S. (2012).Covert Violence in Nursing- A Western Australian Experience. Dissertation Doctorate degree of Philosophy in Public Health. Edith Cowan University. http://ro.ecu.edu.au/theses/455/

Bardakçı, E., \& Günüşen, N. P. (2016). Influence of workplace bullying on turkish nurses' psychological distress and nurses' reactions to bullying. Journal of Transcultural Nursing, 27(2), 166-171.

Berry, P., Gillespie, G., Fisher, B., Gormley, D., \& Haynes, J. (2016). Psychological distress and workplace bullying among registered nurses. Online Journal of Issues in Nursing, 21(3).

Broeder, J. and Donze, A. (2010). The role of qualitative research in evidence-based practice. Neonatal Network, 29(3), 197-202. Retrieved from EBSCOhost 


\section{Aya El Sayed Mahmoud, Karema Ahmed Elsaid, Fawzia farouk Kamel}

Çevik-Akyıl, R., Tan, M., Sarıtaş, S. and Altuntaş, S. (2012). Levels of mobbing perception among nurses in Eastern Turkey. International Nursing Review, 59, 402-408.

Chesler, J. (2014). The impact of workplace bullying on employee morale, job satisfaction and productivity within nonprofit organizations (Doctoral dissertation, Capella University).

Clendon, J., and Walker, L. (2012).Being young: A qualitative study of younger nurses' experiences in the workplace. International Nursing Review, 59, 555-561.

Duru, P., Ocaktan, M., Çelen, ய̈. and Örsal, Ö. (2018). The effect of workplace bullying perception on psychological symptoms: a structural equation approach. Safety and health at work, 9(2), 210-215.

Efe, S. and Ayaz, S. (2010). Mobbing against nurses in the workplace in Turkey. International Nursing Review, 57, 328-334.

Ekici, D. and Beder, A. (2014). The effects of workplace bullying on physicians and nurses. Australian Journal of Advanced Nursing, 31(4), 24-33. Retrieved from EBSCOhost

Elkholy, N. (2015). The Relationship Between Nurses' Organizational Commitment, Their Performance and it is Effect on Patient's Satisfaction at Benha University Hospital, Master Degree, Faculty of Nursing, Benha University ,Egypt

El-Sayed, A. (2015).Workplace Bullying and Absenteeism Rate among Staff Nurses at Mansoura University Hospital. Unpublished master thesis, faculty of nursing, Mansoura University.

Elshahat, M. (2014). The Effectiveness of Implementing Clinical Supervision Model son Head Nurses Performance and Nurses Job Satisfaction at Benha University Hospital, doctoral thesis, Faculty of Nursing, Benha University ,Egypt ,p.20,45,80

Fapohunda, T. (2013). Managing Workplace Bullying. Journal of Human Resource Management. Vol. 1, No. 3, pp. 39-47. Accessed in 21 april 2018 at:http://article.sciencepublishinggroup.com/p df/10.11648.j.jhrm.20130103.11.pdf

Gates, D., Gillespie, G. and Succop, P. (2011).Violence against nurses and its impact on stress and productivity. NURSING ECONOMIC\$, 29(2), 59-67. Retrieved from EBSCOhost

Hee, O. and Kamaludin, N. (2016). Motivation and Job Performance among Nurses in the Private Hospitals in Malaysia. International Journal of Caring Sciences, 9(1).

Hojat, M. and Taheri, L. (2014). Assessment of head nurses quality performance and nursing care quality in critical and general wards of Jahrom university medical sciences hospitals 2012. JULY, 39, 498-501.

Ibrahim, S., El Sayed, R., Attala, M. and Elmezin, N.. (2016). Relationship between head nurses' leadership styles and staff nurses' job performance. IOSR Journal of Nursing and Science (IOSR-JNHS), 5(1), 66-74.

Ikyanyon, D. and Ucho, A. (2013).Workplace bullying, job satisfaction and job performance among employees in 


\section{Effect of Workplace Bullying on Nurses' Job Performance}

federal hospital in Nigeria. European Journal of Business and Management, 5(23), 116-123.

Khalique, M., Arif, I., Siddiqui, M. and Kazmi, S. (2018). Impact of Workplace Bullying on Job Performance, Intention to Leave, OCB and Stress.Pakistan Journal of Psychological Research, 33(1).

Khoshknab, M., Oskouie, F., Najaf, F., Ghazanfari, N., Tamizi, Z., and Ahmadvand, H. (2015). Psychological Violence in the Health Care Settings in Iran: ACross-Sectional Study. Research article Nursing Midwifery Stud. 4(1): e24320.

Lee, M., Kim, H., Shin, D. and Lee, S. (2016). Reliability and validity of the workplace harassment questionnaire for Korean finance and service workers. Annals of Occupational and Environmental Medicine, 18 https://doi.org/10.1186/s40557-016-0133-0

Mete, E. and Sökmen, A. (2016). The influence of workplace bullying on employee's job performance, job satisfaction and turnover intention in a newly established private hospital. International Review of Management and Business Research, 5(1), 65.

Moorhead, S., Johnson, M., Maas, M. and Swanson, E. (2018).Nursing Outcomes Classification (NOC)-E-Book: Measurement of Health Outcomes. Elsevier Health Sciences.

Namie, G. and Namie, R. (2014). Retrieved from Workplace Bullying Institute: http://www.workplacebullying.org/kaplan/

Nwaneri, A., Onoka, A. and Onoka, C. (2016). Workplace bullying among nurses working in tertiary hospitals in Enugu, southeast Nigeria: Implications for health workers and job performance. Journal of Nursing Education and Practice, 7(2), 69.

Olsen, E., Bjaalid, G., Mikkelsen, A., (2017). Work climate and the mediating role of workplace bullying related to job performance, job satisfaction, and work ability: a study among hospital nurses. J. Adv. Nurs. 73 (11), 2709-2719.

Park, J. and Ono, M. (2017). Effects of workplace bullying on work engagement and health: The mediating role of job insecurity. The International Journal of Human Resource Management, 28(22), 3202-3225.

Safarpour, H., Sabzevari, S. and Delpisheh, A. (2018). A Study on the Occupational Stress, Job Satisfaction and Job Performance among Hospital Nurses in Ilam, Iran.Journal of Clinical \& Diagnostic Research, 12(6).

Samir, N., Mohamed, R., Moustafa, E., and Saif, H. (2012). Nurses' attitudes and reactions to workplace violence in obstetrics and gynecology departments in Cairo hospitals. Eastern Mediterranean Health Journal, 18(3), 198-204.

Sharma, J. and Dhar, R. (2016). Factors influencing job performance of nursing staff: mediating role of affective commitment. Personnel Review, 45(1), 161-182.

Shrestha, P. (2016). Challenges and Impact of Transforming Paper-Based Nursing Documentation into Electronic Form: A Study in Nepal (Master's thesis)

Sumathi, G., Kamalanabhan, T. and Thenmozhi, M. (2013). Impact of perceived 


\section{Aya El Sayed Mahmoud, Karema Ahmed Elsaid, Fawzia farouk Kamel}

organisational support on job performance among healthcare professionals. International Journal of Business Innovation and Research, 7(3), 379-391.

Terzioglu, F., Temel, S. and Uslu Sahan, F. (2016). Factors affecting performance and productivity of nurses: Professional attitude, organizational justice, organizational culture and mobbing. Journal of nursing management, 24(6), 735-744.Referanc

Trepanier, S., Fernet, C., Austin, S. and Boudrias, V. (2014). Work environment antecedents of bullying: A review and integrative model applied to registered nurses. International Journal of Nursing Studies, 55, 85-97. doi:10.1016/ j .ij nurstu .2015.10.001

Vessey, J., Demarco, R. and DiFazio, R. (2010). Bullying, harassment, and horizontal violence in the nursing workforce: the state of the science. Annual Review Of Nursing Research,, 28, 133-157.

Yamada, D., Duffy, M. and Berry, P. (2018). Workplace Bullying and Mobbing: Definitions, Terms, and When They Matter. Workplace Bullying and Mobbing in the United States [2 volumes], 1.

Youssif, A., Eid, N. and Safan, S. (2017). Staff Performance Appraisal System and its Relation to Their Job satisfaction and Empowerment: Developing Performance Appraisal Tool. IOSR Journal of Nursing and Health Science Volume 6, Issue 2 Ver. IX PP $17-27$

Zych, I., Ortega-Ruiz, R. and Del Rey, R. (2015). Systematic review of theoretical studies on bullying and cyber bullying: Facts, knowledge, prevention, and intervention. Aggression and Violent Behavior, 23, 1-21. 


\section{تأثير البلطجة في مكان العمل على الأداء الوظيفي للممرضات}

\section{ايه السيد محمود- كريمة احمد السيد- فوزية فاروق كامل}

يعد حدوث البلطجة في مكان العمل من المشكلات الاجتماعية والتنظيمية فى إطار الرعاية الصحية ؛ والتى لها

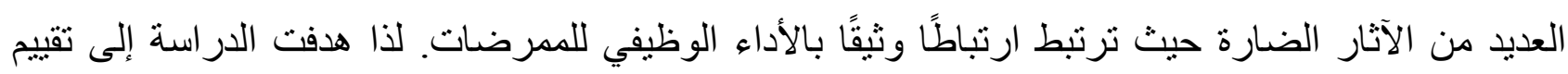

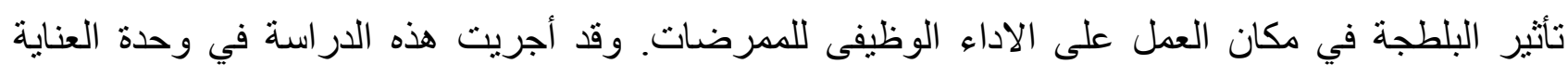

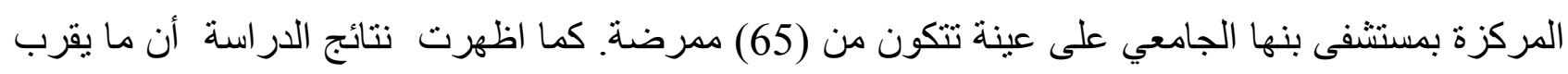

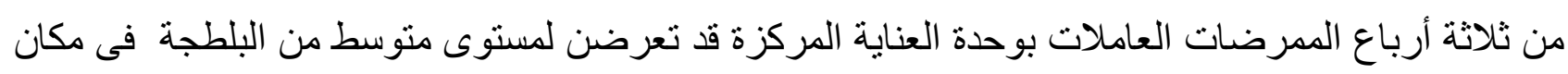

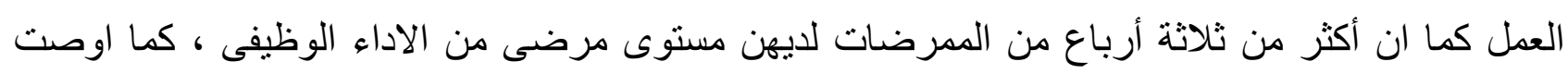

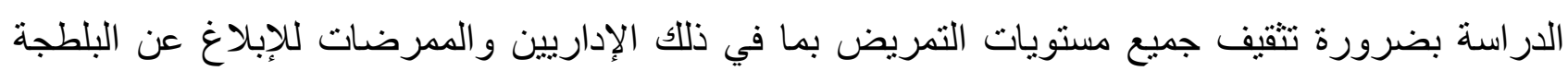
بالاضافة إلى تطوير السياسات لمنع البلطجة وايضا أدوات للإبلاغ عن البلطجة ومكافحتها والتي تسمح للممرضات اللاتى تعرضن لمضايقات في مكان العمل للإبلاغ عن حوادث البر البلطجة. 